(vgl. Kapitel 4.5.1). Und schließlich unterstellen einige Autor*innen einen Bedeutungsverlust der Ministerialverwaltung durch Kürzungen sowie Auslagerungen an externe Beratungsunternehmen (Rudzio 2019: 253; Hustedt et al. 2017; Kalagi 2014; Döhler 2012). Derartige Entwicklungen beschränken die Ressourcen der Ministerialverwaltung zweifelsohne und können das Verhältnis von Prinzipal und Agent langfristig verändern.

Strategien: Bürokratisches-Agenda-Setting und strategische Interaktion

Ressourcen stellen die Basis für die Strategien dar, die ministeriellen Akteuren zur Durchsetzung ihrer jeweiligen Interessen zur Verfügung stehen. Um sich diesem Komplex konzeptionell zu nähern, unterscheiden Schnapp/Willner (2013: 250f.) das bürokratische Agenda-Setting von der strategischen Interaktion. Erstgenanntes beschreibt die Möglichkeit, dass Ministerialverwaltungen eigenmächtig Themen auf die politische Tagesordnung bringen. Strategische Interaktion wiederum heißt, dass sie im Prozess der Politikformulierung eigenmächtig gestaltend tätig werden (vgl. auch Bogumil/Jann 2009: 27; Möltgen-Sicking/Winter 2017: 21f.; Schnapp 2004: 39f.; Klenk 2019: 81f.).

Beim bürokratischen Agenda-Setting und der strategischen Interaktion ist das Verhältnis von Ministerialverwaltung und außerparlamentarischen Interessengruppen wichtig. Neben den Regierungsfraktionen stehen nämlich auch Interessengruppen im engen Austausch mit den sie betreffenden Referaten und nehmen dadurch Einfluss auf politische Prozesse (vgl. Jann/Wegrich 2003: 73). Machura (2005: 40) folgend »bestehen symbiotische Beziehungen in Bezug auf wechselseitige Informationsbeschaffung [und] gemeinsame Interessen an der Erhöhung des politischen Gewichts ihres Bereiches« (vgl. Baruth/Schnapp 2015: 245ff.; Mai 2016: 121ff.). Diese sogenannten Fachbruderschaften führen dazu, dass Gesetzentwürfe bereits frühzeitig - und häufig unterhalb des politischen Radars »verbandsfest« gemacht werden (Rudzio 2019: 66; Machura 2005: 40).

\title{
4.7 Zusammenfassung des Analyserahmens
}

Kapitel 4 hat in den Ansatz eigendynamischer politischer Prozesse als Forschungsheuristik eingeführt und die fünf Erklärungsfaktoren »Instrumente und InstrumentenAlternativen «, »Problemstrukturen«, »situative Aspekte«, »Institutionen« sowie »Akteure und ihre Handlungen« theoretisch ausgearbeitet. Die Interdependenzen zwischen diesen Faktoren sowie die zufälligen Entwicklungen innerhalb einzelner Faktoren werden über das Konzept der Eigendynamik erfasst. Letztlich erzeugt dieses Wechselspiel Rückwirkungen auf die tatsächlich durchgesetzten Policies und damit auf das, was für den Untersuchungsgegenstand des barrierefreien bzw. -reduzierten Wohnraums erklärt werden soll.

Abbildung 9 veranschaulicht die dafür vorgenommenen theoretischen Schwerpunktsetzungen. So wurden die Instrumente und Instrumenten-Alternativen in regulative, ökonomische, kooperative und informationelle Instrumente unterteilt und damit definiert, über welche Steuerungsmöglichen politische Entscheider*innen überhaupt verfügen. Mithilfe dieser Typologie lässt sich nicht nur der bestehende Policy-Mix zum barrierefreien bzw. -reduzierten Wohnraum operationalisieren, sondern auch 
Instrumenten-Alternativen identifizieren: Welche Alternativen werden als ergänzend oder ersetzend zum bestehenden Policy-Mix von einzelnen politischen Akteuren ins Feld geführt? Die erfolgreiche Umsetzung einer Instrumenten-Alternative wird dabei durch die dominierenden Problemstrukturen beeinflusst. Diese unterliegen der subjektiven Deutung einzelner Akteure, wobei etwa Behindertenverbände mutmaßlich andere Interpretationen vornehmen als die Wohnungs- und Bauwirtschaft. Inwieweit handelt es sich beim barrierefreien bzw. -reduzierten Wohnraum um einfache, komplexe oder sogenannte wicked Probleme (vgl. Roberts 2000: 1; Böcher/Töller 2019: 105f.)? Dies ist ebenso zu prüfen wie die Frage, ob situative Aspekte bestimmten InstrumentenAlternativen Möglichkeitsfenster eröffnen und einer Policy damit zur Durchsetzung verhelfen oder hingegen als Unmöglichkeitsfenster die politische Aufmerksamkeit auf andere Themen lenken.

Abbildung 9: Ansatz eigendynamischer politischer Prozesse mit theoretischer Schwerpunktsetzung

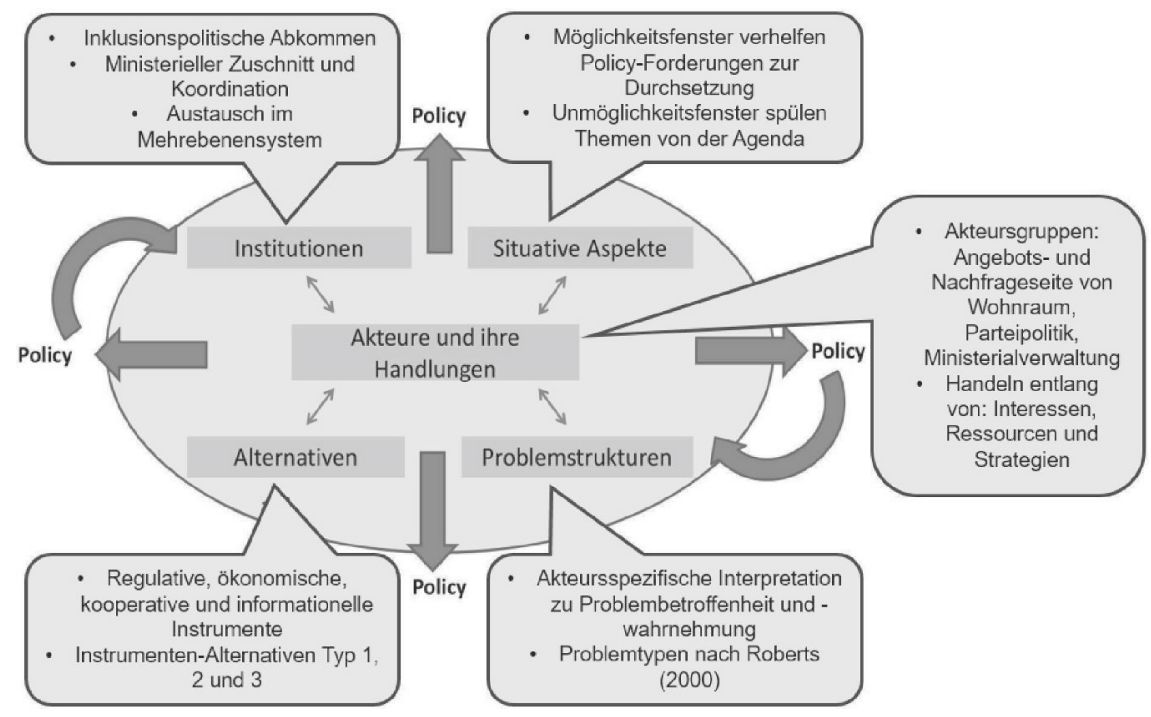

Unter Institutionen interessiert erstens die Relevanz inklusionspolitischer Abkommen, da bereits die Problemstrukturen zum barrierefreien bzw. -reduzierten Wohnraum auf deutliche Schnittstellen zur Sozial- und speziell zur Inklusionspolitik hinweisen. Direkt damit zusammen hängt zweitens der ministerielle Zuschnitt in einem Bundesland. Dieser definiert nicht nur ressortspezifische Handlungsspielräume, sondern beeinflusst auch die Politikintegration zwischen Bau- und Sozialressort. Und drittens interessiert, inwieweit trotz der landespolitischen Zuständigkeiten im Bauordnungsrecht und der sozialen Wohnraumförderung auch Austauschformate zwischen den föderalen Ebenen die politischen Prozesse in einzelnen Bundesländern prägen.

Die Akteure und ihre Handlungen sind als fünfter und letzter AEP-Erklärungsfaktor nach Böcher/Töller (2012: 190) besonders erklärungsmächtig, da sich über die Akteu- 
re die einzelnen anderen Erklärungsfaktoren gegenseitig beeinflussen. So können Akteure beispielsweise situative Aspekte als Möglichkeitsfenster zur Durchsetzung präferierter Policies nutzen, sie können Problemstrukturen umdeuten oder neue Instrumenten-Alternativen auf die politische Agenda setzen - all dies unter der Voraussetzung, dass sie über ausreichende Ressourcen zur Durchsetzung ihrer Interessen verfügen. Für den barrierefreien bzw. -reduzierten Wohnraum wurden die Akteure dreigeteilt in außerparlamentarische Interessengruppen der Angebots- und Nachfrageseite nach Wohnraum, in parteipolitische Akteure sowie die Ministerialverwaltung. Die Interessen, Ressourcen und Strategien dieser Akteure sind dabei wiederum wesentlich vom institutionellen Rahmen abhängig, was die Interdependenz der einzelnen AEP-Erklärungsfaktoren weiter unterstreicht.

Auf Basis dieser theoretischen Ausarbeitung erläutere ich im weiteren Verlauf das methodische Vorgehen meines Forschungsvorhabens (Kapitel 5), um daraufhin die Erklärungskraft der einzelnen Erklärungsfaktoren sowie ihrer Wechselwirkungen in der empirischen Analyse zu überprüfen (Kapitel 6 und 7). 
